\title{
Aluminum Stabilized Superconducting Prototype Cable Development for the mu2e Transport Solenoid
}

\author{
V. Lombardo, G. Ambrosio, D. Evbota, M. Lamm, M. Lopes, T. Page, P. Fabbricatore, R. Musenich
}

\begin{abstract}
The Fermilab Mu2e experiment seeks to measure the rare process of direct muon to electron conversion in the field of a nucleus. The experiment makes use of three large superconducting solenoids: the Production Solenoid (PS), the Transport Solenoid (TS), and the Detector Solenoid (DS). The TS is an "S-shaped" solenoid with a warm-bore aperture of half a meter and field between 2.5 and $2.0 \mathrm{~T}$. The three solenoids are based on four different types of Al stabilized NbTi conductors. All the conductors are composed of a Rutherford cable embedded in an aluminum matrix through a conforming process. This paper summarizes the properties and characterization of 3,000 meters of Al-stabilized prototype cable for the Transport Solenoid (TS). The main results of electrical and mechanical tests are presented and discussed for each stage of the cable development process. In addition to measuring the critical current of extracted strands at Fermilab, samples from each piece length were measured at INFN Genoa. Results are compared and discussed.
\end{abstract}

Index Terms - Aluminum stabilized cables, conforming, mu2e, superconducting cables, Transport Solenoid.

\section{INTRODUCTION}

$\mathrm{T}$ HE Mu2e experiment at Fermilab aims at exploring physics beyond the Standard Model by seeking direct muon to electron conversion in the field of a nucleus. The experiment makes use of three large superconducting solenoids: the Production Solenoid (PS) with $4.5 \mathrm{~m}$ length, $1.5 \mathrm{~m}$ warm-bore aperture, and $4.6 \mathrm{~T}$ peak field on axis; the Transport Solenoid (TS) with $13.4 \mathrm{~m}$ length, $0.5 \mathrm{~m}$ warm-bore aperture, and $2.5 \mathrm{~T}$ peak field on axis and the Detection Solenoid (DS) with $10.9 \mathrm{~m}$ length, $1.9 \mathrm{~m}$ warm-bore aperture, and $2 \mathrm{~T}$ peak field on axis [1] [2]. To build this set of large magnets, a total of four different Aluminum stabilized superconducting cables have been designed (TS, PS, DS1 and DS2). For the Transport Solenoid, the wire and Rutherford cable designs are based on the conductor used for the BELLE detector solenoid [3] at KEK. The TS cable operating current is $1730 \mathrm{~A}$, whereas the peak field on TS coils is $3.4 \mathrm{~T}$; this allows generous temperature and critical current margins for safe operation [4]. A total of 3,000 meters of prototype TS cable were ordered and completely manufactured. In the following the main results of this development process are summarized and discussed.

This work is supported in part by FRA under DOE Contract DE-AC02$07 \mathrm{CH} 11359$.

V. Lombardo, G. Ambrosio, D. Evbota, M. Lamm, M. Lopes and T. Page are with Fermi National Accelerator Laboratory, Batavia, IL 60510 USA (V. Lombardo email: lombardo@fnal.gov). P. Fabbricatore and R. Musenich.are with INFN Genoa, via Dodecaneso, 33, 16146, Genoa, Italy.

\section{TRANSPORT SOLENOID SC STRAND}

The TS strand (Table I) is a multi-filamentary wire with a nominal diameter of $0.67 \mathrm{~mm}$ featuring a minimum $I_{c}$ of $494 \mathrm{~A}$ at $4.22 \mathrm{~K}, 5 \mathrm{~T}$, corresponding to a $J_{c}$ of $2,800 \mathrm{~A} / \mathrm{mm}^{2}$, which is achievable on an industrial scale. A total of two billets were produced by Hitachi Cable for an overall length of $80,000 \mathrm{~m}$.

TABLE I

TRANSPORT SOLENOID STRAND MAIN PARAMETERS

\begin{tabular}{ll}
\hline \hline \multicolumn{1}{c}{ Quantity } & \multicolumn{1}{c}{ Specification } \\
\hline Standard Grade NbTi & $\mathrm{Nb} 47 \pm 1 \mathrm{Wt} \% \mathrm{Ti}$ \\
Strand diameter & $0.67 \pm 0.005 \mathrm{~mm}$ \\
(Cu + Barrier) $: \mathrm{NbTi}$ & $1.0 \pm 0.05$ \\
Filament diameter & $\leq 30 \mu \mathrm{m}$ \\
Number of filaments & 342 \\
Filament twist pitch & $15 \pm 2 \mathrm{~mm}$ (LHS) \\
Copper RRR & $\geq 150$ \\
$I_{c}$ at 5T, 4.22K & $\geq 494 \mathrm{~A}$ \\
$\mathrm{n}$-Value at 5T, 4.22K & $\geq 30$ \\
\hline
\end{tabular}

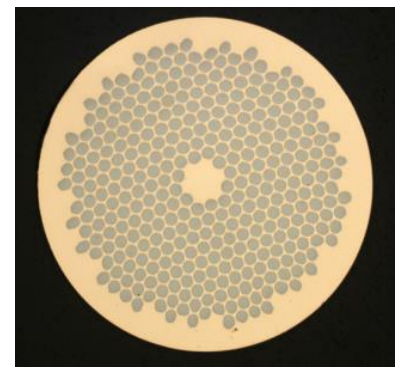

Fig. 1. Cross-section of a Transport Solenoid NbTi virgin strand

Thorough testing was performed both at the Vendor site and at Fermilab to insure the wire met all the specifications. This included $I_{c}$, (Fig. 2-3), n-Values, $\mathrm{Cu} \mathrm{RRR}, \mathrm{Cu} / \mathrm{SC}$ ratio, filament diameter, spacing, and twisting, continuous wire size and ovality as well as eddy current scans.

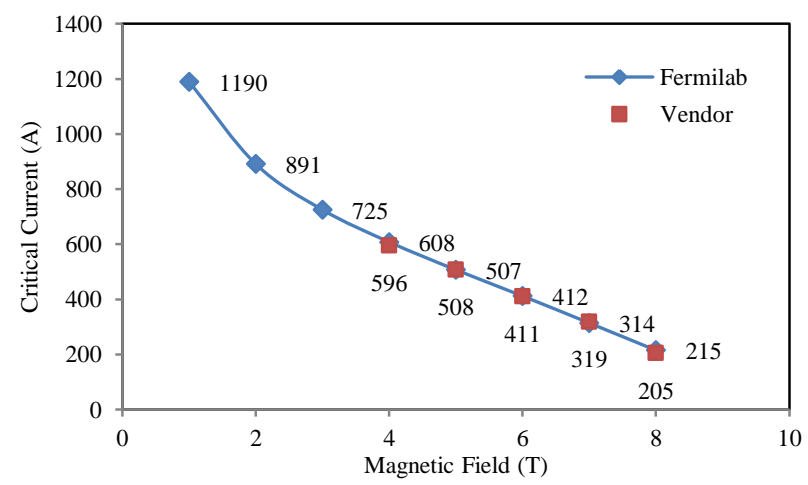

Fig. 2. Typical comparison of $I_{c}$ values measured on TS virgin strands. 


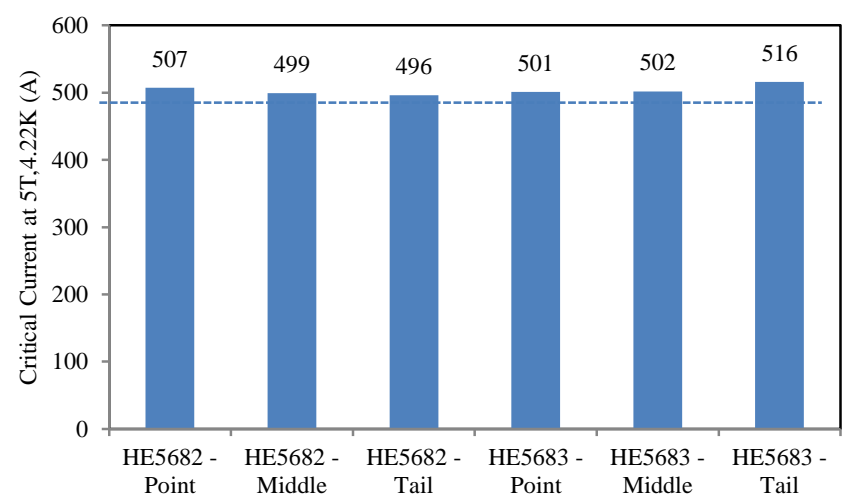

Fig. 3.I $I_{c}$ data collected from beginning, middle and end sections of each billet.

Correlation between Fermilab and vendor's data was found to be extremely good across the spectrum (Fig.2); all the strands exceeded the requirements and were accepted on schedule.

\section{TRANSPORT SOLENOID RUTHERFORD CABLE}

After being accepted, the SC strands were shipped to New England Wire Technologies, to be cabled into two continuous piece-lengths for a total of 5,440 meters. Table II summarizes the main features of the TS Rutherford cable design and Fig. 4 shows a detail of the cable cross-section.

TABLE II

TRANSPORT SOLENOID RUTHERFORD CABLE MAIN PARAMETERS

\begin{tabular}{ll}
\hline \multicolumn{1}{c}{ Quantity } & \multicolumn{1}{c}{ Specification } \\
\hline Number of strands & 14 \\
Cable width & $4.79 \pm 0.01 \mathrm{~mm}$ \\
Cable thickness at $5 \mathrm{kPsi}$ & $1.15 \pm 0.006 \mathrm{~mm}$ \\
Transposition Length & $52 \pm 3 \mathrm{~mm}$ \\
Lay Direction & Right \\
Extracted strand $I_{c}$ at $5 \mathrm{~T}, 4.22 \mathrm{~K}$ & $\geq 469 \mathrm{~A}$ \\
$\mathrm{n}$-Value at 5T, 4.22K & $\geq 30$ \\
Copper RRR & $\geq 150$ \\
Residual Twist & $\leq 45 \mathrm{deg}$ \\
\hline
\end{tabular}

The cabling tooling and process were first tested on a short length of cable, which was shipped to Fermilab for approval. The short length was tested, among other things, for critical current, RRR, broken filaments, mechanical stability and residual twist. Cable dimensions were continuously monitored by New England Wire Technologies using a CMM and calibrated via offline 10-stack measurements. Strands were appropriately marked before being cabled and properly mapped within the cable cross-section to make sure that wires from each billets were cabled, extracted, tested and compared to the relative $I_{c}$ measured on virgin wires. The critical current degradation was found to be less than $2 \%$, whereas a $5 \%$ was accounted for during the cable design.

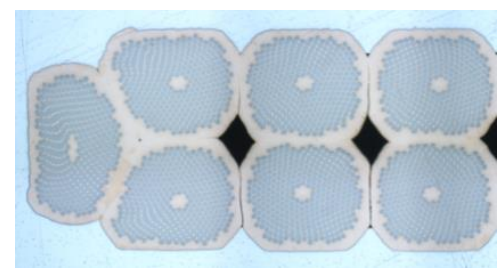

Fig. 4. Detail of the TS Rutherford cable cross-section.
The short length of Rutherford cable exceeded all the cable specifications with margin, so the full length was commissioned. Short samples from the beginning and end of each piece length were shipped to Fermilab for testing before the cable was accepted. During this phase only, all the cold measurements were performed exclusively by Fermilab. Fig. 5 summarizes the critical current results, showing values consistently above specifications. Both piece-lengths were accepted and shipped back to Hitachi Cable for the final conforming phase.

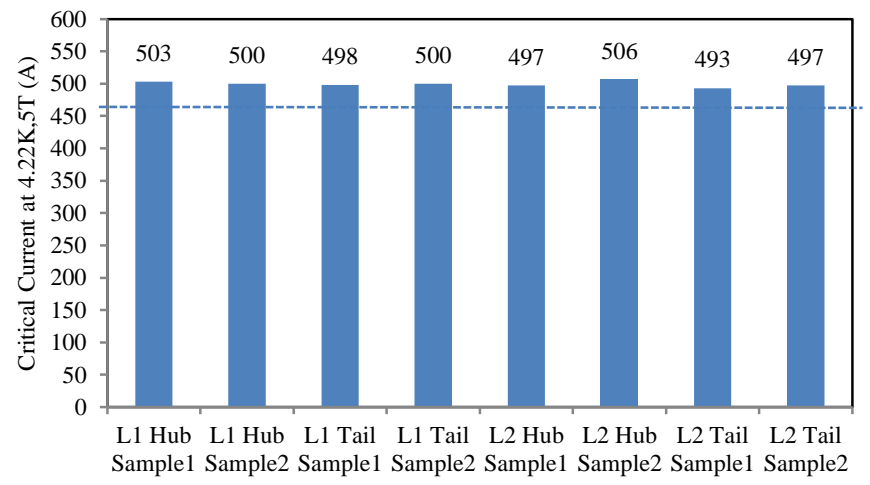

Fig. 5. Summary of $I_{c}$ data collected from wire extracted from both ends of each continuous piece-length of TS Rutherford Cable.

\section{TRAnSPORT Solenoid Aluminum Stabilized CABle}

The design of the Al-stabilized cable was performed taking into account the magnetic, electrical, thermal and mechanical properties and requirements of the TS coil packages [4]. The final cable cross-section is shown in Fig. 6, while the design parameters and the results from the prototype campaign are summarized in Table III.

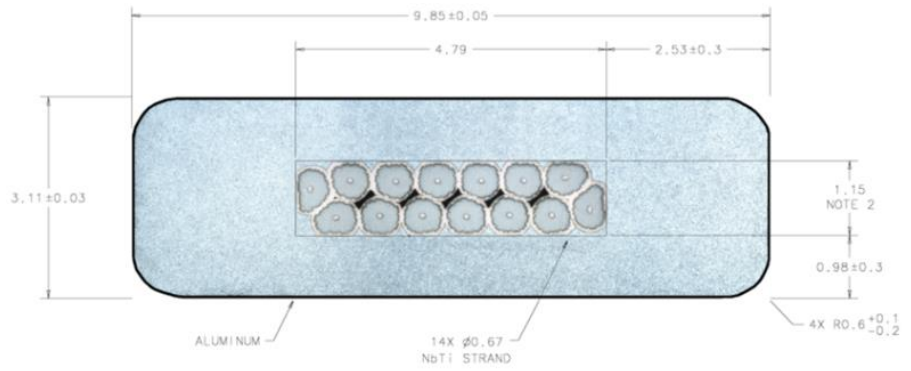

Fig. 6. Cross-section of the Transport Solenoid Al-stabilized cable

TABLE III

TRANSPORT SOLENOID AL-STABILIZED CABLE MAIN PARAMETERS

\begin{tabular}{lll}
\hline \multicolumn{1}{c}{ Quantity } & \multicolumn{1}{c}{ As designed } & \multicolumn{1}{c}{ As procured } \\
\hline Aluminum Stabilizer & $99.998 \%$ & $99.998 \%$ \\
Al RRR before conforming & $\geq 1500$ & $\geq 1700$ \\
Cable width (bare) at RT & $9.85 \pm 0.05 \mathrm{~mm}$ & Within tolerances \\
Cable thickness (bare) at RT & $3.11 \pm 0.03 \mathrm{~mm}$ & Within tolerances \\
Cable $I_{c}$ at $5 \mathrm{~T}, 4.22 \mathrm{~K}$ & $\geq 5900 \mathrm{~A}$ & $5950-6300 \mathrm{~A}$ \\
$\mathrm{n}$-Value at 5T, 4.22K & $\geq 30$ & $38-45$ \\
Copper RRR & $\geq 100$ & $100-104$ \\
Aluminum RRR after cold-work & $\geq 800$ & $925-1160$ \\
Al 0.2\% yield strength at RT & $\geq 30 \mathrm{MPa}$ & $45-56$ \\
Al 0.2\% yield strength at $4.2 \mathrm{~K}$ & $\geq 40 \mathrm{MPa}$ & $74-84$ \\
Al-Cu Shear Strength at RT & $\geq 20 \mathrm{MPa}$ & $35-46$ \\
\hline
\end{tabular}


Three unit lengths for a total of 3,000 meters were manufactured by Hitachi Cable by conforming high purity Aluminum around the Rutherford cable produced by New England Wire Technologies. The cable pre-heating, line speed and conforming temperatures were optimized to achieve a good bonding between the $\mathrm{Al}$ stabilizer and the $\mathrm{Cu}$ matrix while retaining as much $I_{c}$ as possible. After the conforming phase, the cable needs to be cold-worked in order to achieve the mechanical requirements for the Al-stabilizer. This process needed careful optimization in order to attain the right balance between yield and RRR of the stabilizer. For TS, this was accomplished by applying a 9\% overall area reduction to the cable cross-section. Results summarized in Fig. 7-10 show how all the cold-worked TS cable piece lengths met all the electrical and mechanical requirements.

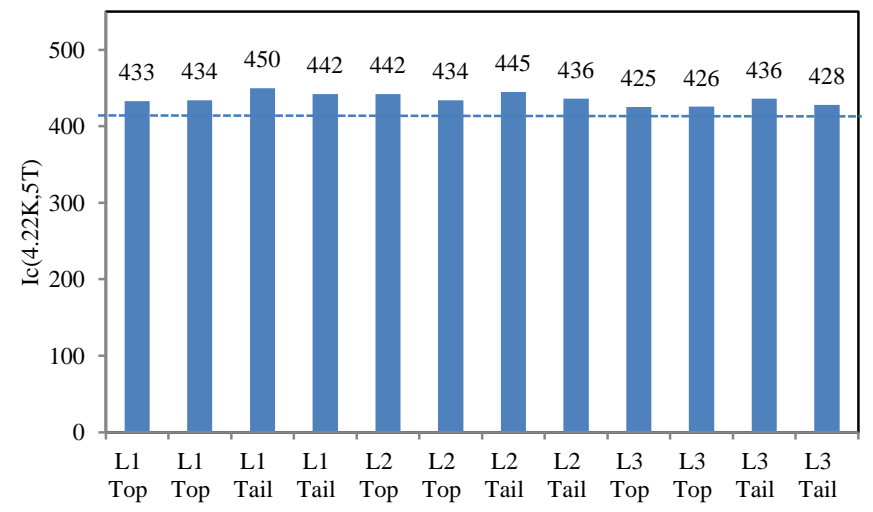

Fig. 7. Summary of $I_{c}$ measurements performed on wires extracted from both ends of each Al-stabilized cable unit length after cold-work. Strands are extracted by chemically etching the Al-stabilizer.
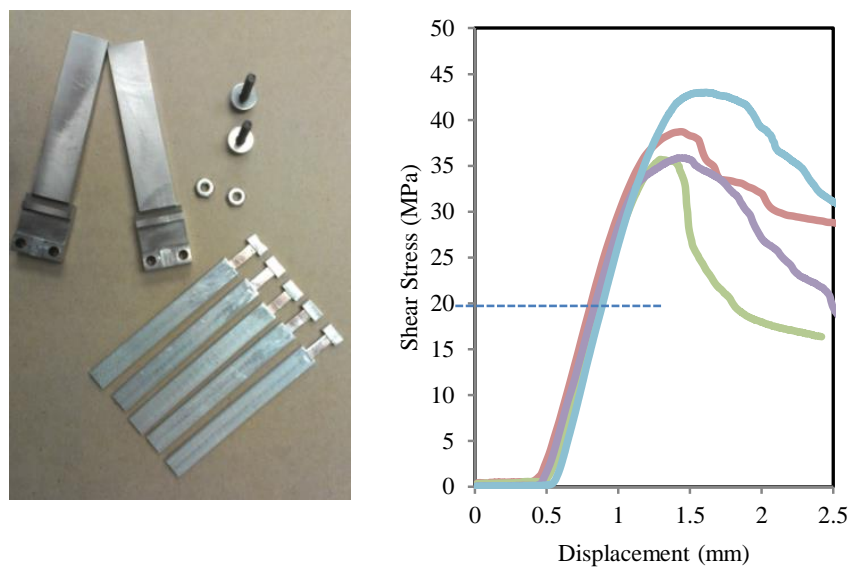

Fig. 8. Left: TS samples with partially removed Aluminum stabilizer to check the quality of the bonding. Right: Example of shear stress curves between the Aluminum stabilizer and the TS Rutherford cable. Measurements were performed at room temperature.

The Al-Cu bonding properties were checked using coldworked cable short samples cut from both ends of each continuous piece-length. The aluminum was locally machined in order to expose the Rutherford cable (Fig. 8, left) while a 5 $\mathrm{mm}$ aluminum cap was left intact. A clamp specifically designed for the TS conductor was used to pull the cap via an Instron ${ }^{\circledR}$ machine to measure the ultimate shear stress between the Aluminum stabilizer and the superconducting cable (Fig.8, right). The shear stress measured on all the TS prototype cable lengths exceeded the specification of $20 \mathrm{MPa}$ at room temperature with a sizeable margin, showing values between 35 and $46 \mathrm{MPa}$. In addition to destructive pull-tests, the quality of $\mathrm{Al}-\mathrm{Cu}$ bonding was investigated by polishing cable cross-sections from both ends of each piece length to insure the presence of a diffusion layer between the Aluminum stabilizer and the copper matrix in the superconducting wires. The cross-sections analyzed for the TS prototype showed an intermetallic diffusion layer thickness between 1.5 and $2 \mu \mathrm{m}$, which is representative of a good bonding.

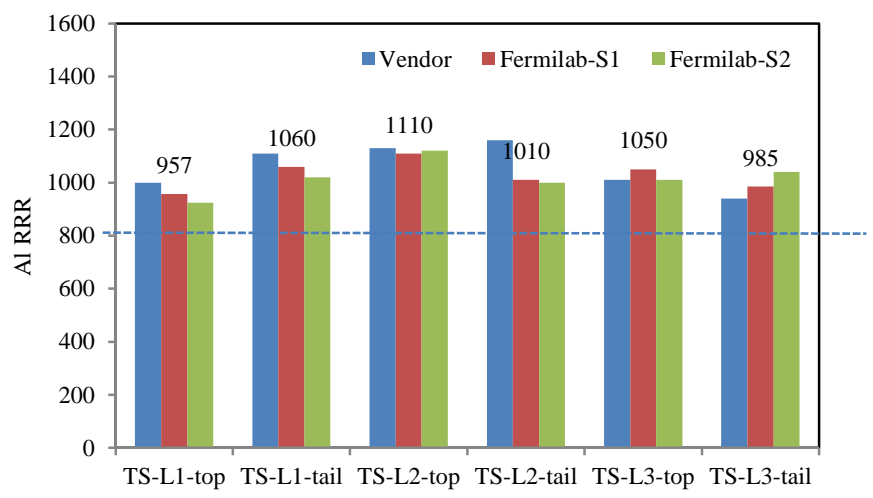

Fig. 9: Summary of RRR measurements taken from Al stabilizer samples extracted from the cable cross-section via EDM.

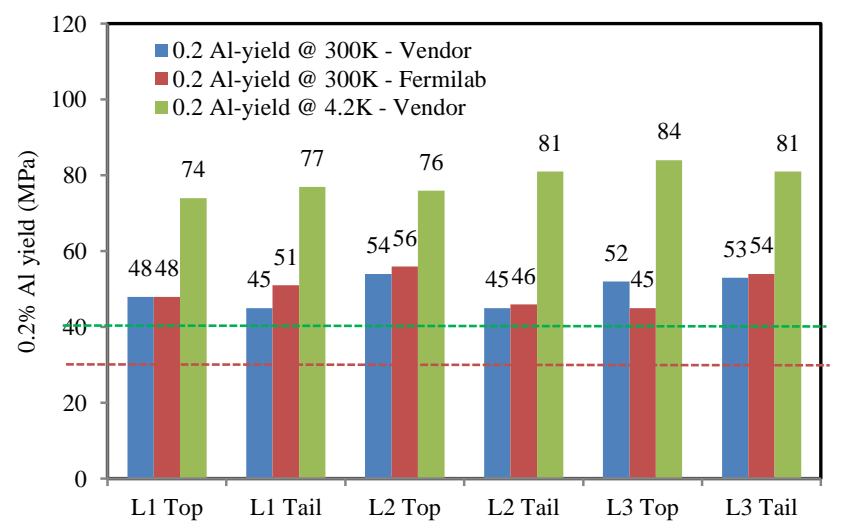

Fig. 10. Summary of $0.2 \%$ Al-yield measurements taken from Al stabilizer samples extracted from the cable cross-section via EDM. Measurements were performed both at room temperature and liquid helium temperature. In both cases, the specifications are met with margin.

\section{Cable Critical Current Measurements}

The critical current measurement of full TS conductor samples (including the Al stabilization) was performed using the facility Ma.Ri.S.A. at INFN Genoa, via the methods developed for characterizing the CMS conductor [5]. The facility is based on a superconducting solenoid providing a $6 \mathrm{~T}$ magnetic field [6] in a $500 \mathrm{~mm}$ bore hosting a cryostat with a $440 \mathrm{~mm}$ diameter. The samples are closed in a low resistance loop and the current is induced using the direct transformer method [7]. The TS conductor samples are bent on their higher inertia axis (hard way bent) and the two ends are overlapped 
and indium soldered to form a continuous ring. The resulting ring is finally mounted inside an aluminum alloy (5083) sample holder as shown in Fig. 11.

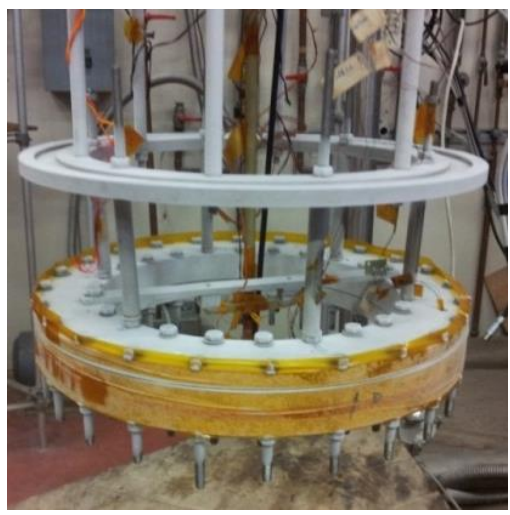

Fig. 11: Sample holder for in-field $I_{c}$ cable testing at INFN Genoa.

The current is induced in the sample ring using the background magnet as the primary coil and the sample as the secondary one. The magnetic field is applied perpendicularly to the wide face of the cable. When performing the critical current measurement of high current conductor one has to face the problem related to the definition itself of the critical magnetic field, because the field generated by the conductor may be a not negligible fraction of the total applied field. As an example, Fig. 1 shows the self-field distribution on the TS conductor when a current of $10 \mathrm{kA}$ flows through it. In [8] a simple and reliable method was discussed for assigning a critical field to a $I_{c}$ measurements on large flat cable with magnetic field applied normally to the wide face. Shortly, the average component of the magnetic field normal to the wide face $B_{z_{-} \text {self }}$ is evaluated on the two strands exposed to the highest magnetic field and summed to the external magnetic field $\mathrm{B}_{\mathrm{ext}}$.

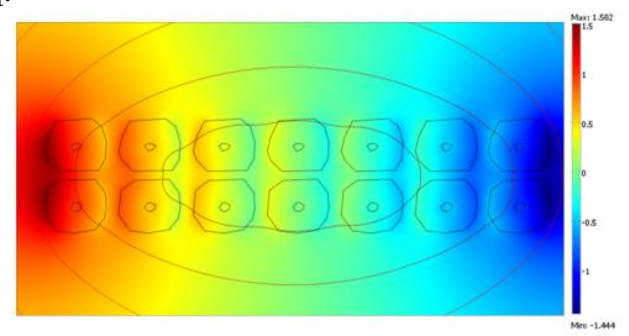

Fig. 12. Self-field distribution on NbTi filaments within the cable crosssection when the cable is powered with 10kA.

The resulting field $B_{\text {app }}=B_{z_{z} \text { self }}+B_{\text {ext }}$ is taken as the applied magnetic field. Basically one can see that only the two considered strands are contributing to the voltage drop along the conductor. Using this approach, the measured $I_{c}\left(\mathrm{~B}_{\text {ext }}\right)$ can be directly compared with the critical current measured on extracted strands without any self-field correction. This method is alternative to the peak field correction consisting in summing up the peak self-field to the external field for both the cable and the strand. Fig. 13 shows a comparison between $I_{c}$ data collected from Al-stabilized cable at INFN and data collected from extracted wire from the same cables at
Fermilab. The results are found to be in good agreement.

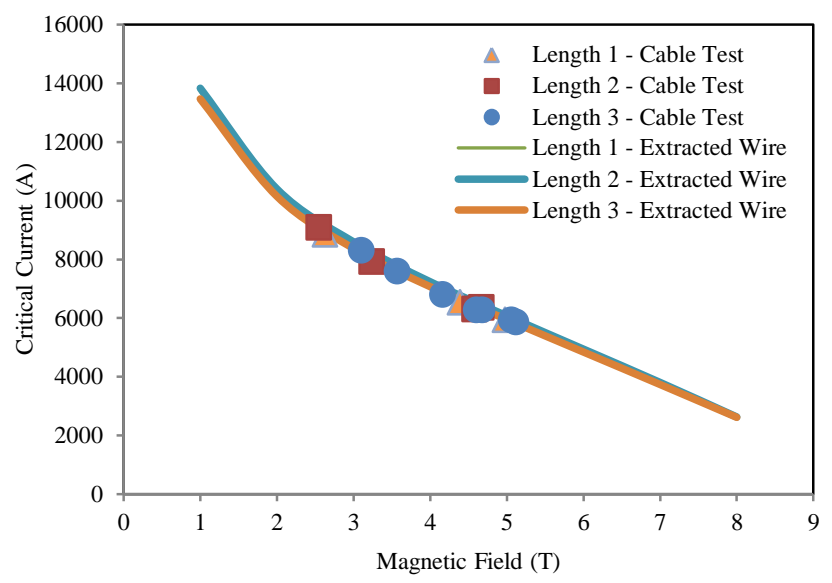

Fig. 13. Summary of $I_{c}$ data from Al-stabilized cables and comparison with data collected from extracted wires from the same cables.

\section{CONCLUSIONS}

The prototype phase for has been successfully completed on schedule with the delivery of 3,000 meters of TS cable. A TSstyle prototype coil is currently being manufactured in industry using two of the three piece lengths. The production phase has recently been launched, with the goal of producing $700 \mathrm{~km}$ of superconducting wire and $44 \mathrm{~km}$ of TS Alstabilized cable needed to build the entire Transport Solenoid. The whole experiment will need over $70 \mathrm{~km}$ of Al-stabilized cable to be completed.

\section{ACKNOWLEDGMENT}

The authors wish to thank the team at the Superconducting Cable R\&D Lab at Fermilab for the invaluable help and support with short sample preparation and testing; Marianne Bossert and Pei Li (Fermilab) for the support with metallography as well as Sergio Burioli (INFN Genoa) for the extensive help in cable sample preparation. Finally, the teams at Hitachi Cable America (http://www.hca.hitachi-cable.com), SH copper (http://www.shcopper.com) and New England Wire Technolgies (http://www.newenglandwire.com).

\section{REFERENCES}

[1] Mu2e Collaboration, "Mu2e Conceptual Design Report", available online at: http://arxiv.org/abs/1211.7019

[2] M. Lamm, et al., "Solenoid Magnet System for the Fermilab Mu2e Experiment", IEEE Trans. Appl. Supercond. 22, 2012.

[3] I.L. Horvath, et al., "Aluminum Stabilized Superconductor for the BELLE Detector at KEK-B", Proceedings of fifteenth International Conference on Magnet Technology, p. 1044 - 1047.

[4] G. Ambrosio, et al. "Challenges and Design of the Transport Solenoid for the Mu2e Experiment at Fermilab“IEEE Trans. Appl. Supercond, 24, 2014

[5] M. Greco, P. Fabbricatore, R. Musenich, IEEE Trans.Appl. Supercond. 12 (2002) 381.

[6] P. Fabbricatore, A. Parodi, R. Parodi, and R. Vaccarone, Proc. ICEC12, Butterworth, 1988, pp. 879-882.

[7] P. Fabbricatore and R. Musenich, "Handbook of Applied Superconductivity, B. Seeber, Ed. Bristol: IOP Publishing, 1998, pp. 325-343

[8] M. Greco, P. Fabbricatore, S.Farinon, R. Musenich Physica C 401 (2004) 124-128 